\title{
1 Analysis of gene functions in Maize chlorotic mottle virus
}

2 Kay Scheets*

3 *Corresponding author:

4 K. Scheets, Department of Plant Biology, Ecology, and Evolution, 301 Physical Sciences, Oklahoma 5 State University,

6 Stillwater, OK, USA, 74078-3013. Phone: 405-744-5559; Fax: 405-744-3285;

7 e-mail: kay.scheets@okstate.edu 


\section{Abstract}

2 Gene functions of strains of Maize chlorotic mottle virus, which comprises the monotypic genus

3 Machlomovirus, have not been previously identified. In this study mutagenesis of the seven genes

4 encoded in maize chlorotic mottle virus (MCMV) showed that the genes with positional and sequence

5 similarity to their homologs in viruses of related tombusvirid genera had similar functions. p50 and its

6 readthrough protein p111 are the only proteins required for replication in maize protoplasts, and they

7 function at a low level in trans. Two movement proteins, p7a and p7b, and coat protein, encoded on

8 subgenomic RNA1, are required for cell-to-cell movement in maize, and p7a and p7b function in trans.

9 A unique protein, p31, expressed as a readthrough extension of p7a, is required for efficient systemic

10 infection. The 5' proximal MCMV gene encodes a unique $32 \mathrm{kDa}$ protein that is not required for

11 replication or movement. Transcripts lacking p32 expression accumulate to about 1/3 the level of wild

12 type transcripts in protoplasts and produce delayed, mild infections in maize plants. Additional studies

13 on p32, p31 and the unique amino-terminal region of p50 are needed to further characterize the life

14 cycle of this unique tombusvirid.

17 Key words: Machlomovirus, Tombusviridae, RNA virus replication, gene function, viral movement, 18 overprinted gene 


\section{Introduction}

Maize chlorotic mottle virus is the only member of the genus Machlomovirus in the family

Tombusviridae (Rochon et al., 2011). The host range of maize chlorotic mottle virus (MCMV) is restricted to Poaceae, and MCMV is readily infectious by mechanical inoculation. MCMV is part of a synergistic disease called corn lethal necrosis (CLN) (Niblett and Claflin, 1978) or maize lethal necrosis (MLN) (Wangai et al., 2012) when it coinfects with a virus in the family Potyviridae. MCMV levels in MLN plants rise dramatically (Goldberg and Brakke, 1987; Scheets, 1998; Xia et al., 2016) as commonly seen with potyvirid synergisms.

The 5' two-thirds of the 4437 nt viral RNA encode three proteins (Nutter et al., 1989). The first open reading frame (ORF) encodes a $32 \mathrm{kDa}$ protein, p32 which is unique to MCMV (Fig. 1). p50 and its readthrough (rt) protein p111 are related to the highly conserved RNA dependent RNA polymerases (RdRps) encoded by other members of the family Tombusviridae. p50 is similar in size to the ORF1 product of Panicum mosaic virus, genus Panicovirus,(48 kDa) and two other panicoviruses (41-44 $\mathrm{kDa}$ ) which are larger than the related ORF1-encoded proteins (23-37 kDa) of other tombusvirids. p50 has a unique amino-terminal sequence that coincides with most of the p32 ORF overlap (residues 1283).

All ORFs in the 3' third of the genome are expressed from subgenomic RNA1 (sgRNA1) (Scheets, 2000). The first ORF encodes a $7.2 \mathrm{kDa}$ peptide (p7a) and suppression of its stop codon produces a $31 \mathrm{kDa}$ protein, p31 (Scheets, 2000). The carboxy-terminus of p7a exhibits sequence similarity to movement protein 1 (MP1) found in tombusvirid genera that encode two or more small MPs (Rochon et al., 2011). A small ORF with no methionine codons ( 8 kDa coding capacity) following the p7a ORF was later identified by comparison to carmovirus sequences (Riviere and Rochon, 1990), and the encoded peptide is most closely related to MP2 from panicoviruses (Scheets et al., 2015). MP1 and MP2 are required for cell-to-cell movement in viruses from four tombusvirid 
1 genera (Genoves et al., 2006; Hacker et al., 1992; Molnar et al., 1997; Pantaleo et al., 1999; Turina et

2 al., 2000; Yuan et al., 2006) and an unassigned tombusvirid (Castaño et al., 2009; Scheets et al., 2015).

3 The carboxy-terminal extension of the $31 \mathrm{kDa}$ protein is unique. The second AUG of sgRNA1 begins

4 the $25 \mathrm{kDa}$ coat protein (CP) ORF (Scheets, 2000). A 337 nt noncoding RNA (sgRNA2) accumulates

5 in MCMV infected protoplasts and plants (Scheets, 2000), although it is not known whether it is a true sgRNA or a structure-protected degradation product as determined for red clover necrotic mosaic virus

7 (Iwakawa et al., 2008).

This project was undertaken to determine the functions of the MCMV-encoded proteins. As

well as being noninfectious to dicots, MCMV is not amenable to expression of a reporter gene since attempts to replace MCMV coding regions or fuse a reporter to the CP ORF were lethal (unpublished results). Complicating analyses, no good local lesion host for MCMV is available. Therefore, functions of all MCMV ORFs were analyzed by comparing the ability of mutant and wild type (WT) transcripts to replicate in maize protoplasts and to infect and spread in maize plants.

\section{Materials and methods}

\subsection{Construction of mutant cDNAs and other plasmids used in the study}

In vitro mutagenesis (Kunkel et al., 1987) of pMCM41 (Scheets et al., 1993) with

oligonucleotides listed in Table S1 was used to make p32KO, p50-Aug, p111rt, and p50-2stop, while p7bMet, p7bKO, p7bpTer, and p7bQ12N were made using a Gene Editor kit (Promega Corp., Madison WI). Construction of pCP-Aug, p7apTer, p7art, and p7a/CP was described previously (Scheets, 2000). $\Delta$ GDD was serendipitously produced during p7apTer construction and is missing nt 2244-2675 with the same 3' changes as p7apTer. Plasmids were selected by restriction digestion and/or sequencing (Scheets, 2000). Automated dideoxy sequencing was performed on an ABI instrument, and sequence data was analyzed using Sequencher (Gene Codes Corp., Ann Arbor, MI).

2.2 Black Mexican Sweet (BMS) maize protoplast inoculations 
2 For each experiment, equal aliquots $\left(1-3 \times 10^{6}\right)$ of protoplasts were inoculated with equal amounts of

3 mutant or pMCM41 transcript RNAs (7.5-15 $\mu \mathrm{g})$ synthesized and purified as described previously

4 (Scheets, 2000). For coinoculations of p111rt and p50-2stop transcripts, both RNAs were added at the

5 concentration used in single inoculations. Samples were collected at 0, 24, and 48 hours post-

6 inoculation (hpi). CP detection by protein A sandwich-enzyme linked immunosorbent assay (PAS-

7 ELISA) was described previously (Scheets, 2000). Dilutions of large scale purified MCMV virions

8 (Scheets 1998) quantified spectrophotometrically $\left(6.7 \mathrm{~cm}^{2} \mathrm{mg}^{-1}\right)$ and assayed on the same 96-well plate

9 were used to determine MCMV concentrations by linear regression. Concentrations at 48 hpi were

10 converted to \% WT. Total RNA isolation, electrophoresis, and phosphorimage detection was

11 previously described (Scheets 2000). Genomic RNA (gRNA) bands were quantitated from

12 phosphorimage data and compared to WT levels for experiments that showed equivalent RNA sample

13 loading.

14

\subsection{Plant inoculations and visual symptom assessment}

Maize (var. PH3168) was grown in greenhouses, and supplemental light was added for some experiments during months with short day lengths (Oct-Feb) to provide $13 \mathrm{hr}$ light. At the 3-leaf stage, plants were taken to the laboratory, dusted with carborundum and inoculated on the third leaf with 10 $\mu \mathrm{l}$ containing 8-10 $\mu \mathrm{g}$ transcript in $0.5 \%$ bentonite by three strokes with a gloved finger. Fresh gloves were used for each inoculum. For individual experiments the same amount of RNA was used/plant, and 3-6 seedlings/transcript were inoculated. For coinoculations, RNAs were mixed and added at the concentration used in single inoculations. Inoculated leaves were rinsed with deionized water 5-10 min post-inoculation. Plants were kept in a dark humid chamber overnight before returning them to the greenhouse. Barley seedlings (var. IAR/B/334-2) were grown and inoculated similarly except the second leaf was inoculated and light supplementation was for $14 \mathrm{hr}$. Typically, MCMV systemic 
1 symptoms in PH3168 consist of a mild chlorotic mottle and appear first on leaf 4 or 5 about 4-8 days

2 post-inoculation (dpi). The intensity of WT MCMV symptoms varies with growth conditions, with

3 strongest symptoms produced by high light intensities and warm temperatures $\left(28-31^{\circ} \mathrm{C}\right)$ (Scheets,

4 1998) such that after a week of cloudy weather in a greenhouse, previously observed mild symptoms

5 may become almost invisible. In all 11 maize inoculation experiments, $100 \%$ of plants inoculated with

$6 \quad$ WT transcripts were systemically infected.

\section{$7 \quad 2.4$ CP detection in plants}

For CP detection, inoculated leaves were sampled weekly (7 mm disc, 4-5 mg) (Scheets, 1998) beginning at 7 dpi. At 14 (or 13) dpi, 21 (or 20) dpi, and later time points, newest leaf samples were

10 also collected. For some experiments, final samples were collected from all upper leaves. CP was

11 quantified as for protoplast assays, and CP was converted to $\mu \mathrm{g} / \mathrm{sample}$ before calculating the mean and 12 standard error of the mean.

\subsection{Northern blots of total RNA from plants and RNA dot blots of crude leaf extracts}

Total RNA isolation and northern blots from water-washed inoculated leaves and newest leaves were done as in Scheets (1998). For some experiments duplicate $7 \mathrm{~mm}$ discs from inoculated leaves were washed by briefly vortexing in $0.2 \mathrm{ml} 0.5 \%$ SDS, and the solution was discarded after $1 \mathrm{~min}$. Washed inoculated leaf samples and upper leaf samples were stored at $-80^{\circ} \mathrm{C}$. The discs were ground in $0.2 \mathrm{ml} \mathrm{GSCN}$ Buffer and the crude extracts were prepared for blotting as described (Scheets, 2013). Thirty microliters (1/80 of sample) was loaded onto 96-well dot blots. Dilutions of similarly denatured full length transcripts of pMCM41 (5-50 ng/well) were used as standards.

\subsection{Small-scale MCMV virion/vRNA isolations}

Leaf tissue from each maize plant (0.15-0.4 g inoculated leaf and 0.34-0.5 g of upper leaf) was separately collected 20-30 dpi. Inoculated leaves were washed in 0.5\% SDS, rinsed, and dried before all leaf samples were chopped and stored at $-80^{\circ} \mathrm{C}$. Mini-virion isolations were performed (Scheets, 
1998) and stored at $4^{\circ} \mathrm{C}$. MCMV vRNA was isolated and stored as dry RNA pellets at $-20^{\circ} \mathrm{C}$, then

dissolved in $20-50 \mu \mathrm{L}$ of $50 \%$ formamide or sterile deionized water and stored at $-80^{\circ} \mathrm{C}$ (Scheets, 1998).

\subsection{Northern and dot blot hybridizations}

Dot blot hybridizations were done at $60^{\circ} \mathrm{C}$ using $(-)^{32} \mathrm{P}$-labelled riboprobes made from pMCM602 (5' 1/4 of MCMV genome) under high stringency conditions (Tm-10C) (Scheets, 1998).

Protoplast northern blots were incubated at $60^{\circ} \mathrm{C}$ using $(-)^{32} \mathrm{P}$-labelled riboprobes made from BS-SpeI (3' terminal $253 \mathrm{nt}$ of MCMV) at Tm-20 $\mathrm{C}$ (Scheets, 1998). Washed blots were exposed to either Kodak XAR film with or without a Cronex intensifying screen or a Molecular Imager (Bio-Rad Laboratories, Hercules, CA), which was also used for quantitation.

2.8 RT-PCR amplification from mini-virion $v R N A$ preparations

Reverse transcription was performed at $50^{\circ} \mathrm{C}$ with SuperScript II Reverse Transcriptase (Invitrogen, Carlsbad, CA) following manufacturer's conditions. Reactions contained 1-6 $\mu \mathrm{L}$ of undiluted or diluted vRNA, and either M457c or p25sg RO (Table S1) as primer. PCR amplifications used phosphorylated primers (Table S1) and Vent DNA polymerase (New England Biolabs, Ipswich, MA) following manufacturer's recommendations. Amplified DNA was cleaned using a QIAquick PCR Purification Kit (Qiagen Inc., Valencia, CA), and selected fragments were analyzed by restriction enzyme digestion before agarose gel electrophoresis on $1.5 \%$ agarose gels with a 100 bp DNA ladder (New England Biolabs). PCR products were directly sequenced, and selected PCRs were cloned into SmaI-digested pBluescript SK+ for sequencing of individual DNAs.

\section{Results}

3.1 p50 and its readthrough protein are the only proteins required for replication To determine the functions of each MCMV protein, mutant versions of the WT infectious transcript plasmid pMCM41 were constructed that altered the expression of one or more ORFs, and the 
transcripts from these mutants were tested for their ability to infect protoplasts and plants. The first MCMV AUG was altered in p32KO by introducing an ApaI site (Fig. 1). No full length or truncated p32 can be synthesized from p32KO transcripts since the next three AUGs are in other reading frames.

For p50-AUG transcripts, the next AUG is in the p50 ORF, so an amino-truncated $47 \mathrm{kDa}$ version of this protein can be synthesized. The p50 stop codon was changed to a tryptophan codon in p111rt, and p502stop had a second stop codon added downstream of the p50 stop codon to terminate any synthesis of the p111 readthrough region. A serendipitous plasmid containing a 432 bp deletion that removed the coding region for the GDD active site of RdRp ( $\triangle \mathrm{GDD}$ ) was isolated during construction of p7apTer and served as a nonreplicating control (Fig. 1, Table 1).

Inoculation of BMS protoplasts with mutant and WT transcripts showed that the ORFs for p50 and its readthrough region encode the only viral proteins required for replication (Fig. 2 and data not shown). Protoplasts inoculated with p32KO transcripts synthesized 30\% of the WT level of gRNA and accumulated CP at 34\% of the WT level. Transcripts that expressed the amino-truncated p50 product (p50-AUG) with its corresponding readthrough protein produced 27\% as much gRNA as WT transcripts and accumulated CP at 25\% of the WT level (Fig. 2). Transcripts for p50-2stop, p111rt, and $\Delta$ GDD were not able to replicate when tested individually. These mutants produced no detectable accumulation of CP while long exposures of northern blots showed intact and degraded inoculum (Fig. 2). Additionally, protoplasts coinoculated with p111rt and p50-2stop transcripts showed that transcomplementation occurred at very low levels since gRNA, sgRNA1, and CP accumulated. For two experiments more accurate quantitation was obtained from a second northern blot after electrophoresis of 1/20 as much total RNA from high-replicating samples (WT, p32KO, p50-AUG) with a second aliquot of undiluted total RNA from low signal samples (Fig. 2 and data not shown). In double-inoculation experiments where gRNA accumulated to at least $1 \%$ of the WT level, sgRNA1 was detected and CP accumulated at low levels (6-11\% of WT). These results showed that p50 and its readthrough protein 
1 p111 are the only viral proteins required for viral replication, and that the proteins function at a low

2 level in trans. Removing the $3 \mathrm{kDa}$ amino-terminus of p50 decreased vRNA and CP accumulation

3 about four-fold. Additionally, although p32 was not required for replication, viral RNAs and CP

4 accumulated to higher levels when this protein was present.

5 3.2. None of the proteins expressed from sgRNA1 are required for replication only impair or block expression of p31 and leave remaining ORFs unchanged. Since all 3' ORFs are expressed from sgRNA1, several ORFs were altered by more than one method since it was uncertain how upstream mutations might affect translation of downstream ORFs (Fig. 3).

Mutant transcripts that impaired the expression of p7a and its readthrough protein (p7apTer), changed the p7a stop codon to a Trp codon to allow expression of the $31 \mathrm{kDa}$ readthrough protein without p7a expression (p7art), impaired expression of both p31 and p7b (p7a/CP), or altered the first

Due to the overlap of the sgRNA1 ORFs, it was impossible to introduce mutations that would AUG of the CP ORF (pCP-AUG), can replicate in BMS protoplasts (Scheets 2000). All but pCP-AUG accumulate MCMV RNAs at levels $\geq$ WT and produce immunodetectable CP at 53-75\% of WT inoculations (Scheets, 2000 and data not shown).

New mutants produced for this study include the plasmid p7bKO, which eliminated synthesis of p7b by mutating its CTG start codon, and p7bpTer which added two premature stop codons to the p7b ORF, reducing its size from 64 to 12 residues. In p7bQ12N, expression of the complete $31 \mathrm{kDa}$ readthrough region was eliminated by adding a second stop codon downstream of the p7a stop codon which produced a conservative glutamine $(\mathrm{Q})$ to asparagine $(\mathrm{N})$ substitution in $\mathrm{p} 7 \mathrm{~b}$ at position 12 . The p7b start codon was changed to ATG in p7bMet, and this mutation also changed the overlapping p31 ORF from an alanine codon to an aspartate codon immediately after the p7a stop codon (Fig. 3). The new mutant transcripts were tested in BMS protoplasts, and all were replication competent (Figs. 4 and S1). Comparison of ethidium bromide stained 18S rRNA levels indicated some total RNA 
was lost during RNA isolation of p7bMet and WT samples. When intensities of gRNA bands were compared to ethidium bromide staining intensities, it was found that similar relative levels of gRNAs accumulated in samples inoculated with mutant and WT transcripts. Quantitation of CP at 48 hpi indicated that p7bKO and p7bpTer produced CP at WT levels, while p7bQ12N CP accumulation (87\%) was similar to results for p7apTer, p7art, and p7a/CP (Scheets 2000, Fig. 4 and data not shown). p7bMet produced 7\% as much CP as WT from similar levels of sgRNA1 which resembles pCP-AUG

ELISA signals (6\% of WT) (Fig. 4, Scheets 2000, and data not shown). These results further confirmed that none of the proteins expressed from sgRNA1 were necessary for replication, and mutations that change translatability of upstream sgRNA1 ORFs may alter accumulation of CP.

3.3 p32 is not required for infection or spread in plants but its absence dramatically decreases virus accumulation and symptoms

Replication competent mutant transcripts were inoculated to maize seedlings to investigate the functions of MCMV genes not required for replication. Seven maize inoculation experiments were performed with p32KO, and only in one experiment in which WT transcripts produced very strong symptoms, due to a new clear greenhouse roof and sunny weather, was a mild chlorotic mottle observed on some p32KO-inoculated plants. Systemic symptoms appeared 6-13 days later than WT, and all six plants were ELISA-positive in newest leaves at 14 dpi (Table 1). Low levels of CP and RNA could be detected in some of the inoculated leaves at 7 dpi or later in all experiments, and extremely low levels of $\mathrm{CP}$ were occasionally detected in the newest leaf at $14 \mathrm{dpi}$ or later in asymptomatic experiments. Systemic infections were confirmed in two-thirds of p32KO-inoculated plants of two further experiments. To assess relative virus concentrations in the whole plant, samples were collected from inoculated leaves and the middle of all upper leaves of plants inoculated with p32KO or WT for CP quantitation at the final time point (26 or 28 dpi). For one experiment p32KOinfected plants accumulated about 1/14 the amount of CP as WT-infected plants, with leaves at 
1 particular positions showing 7-fold to 21-fold less CP than the same positions in WT-inoculated plants

2 (Fig. 5). Similar but less dramatic results were obtained for the other experiment (data not shown).

3 Virions and vRNA were isolated from the inoculated leaves and selected upper leaves of p32KO- and

4 WT-infected plants from experiments with confirmed systemic infections. Restriction digest analyses

5 of RT-PCR fragments showed that all of the p32KO samples were cut by ApaI completely or almost

6 completely (Fig. S2B), indicating that the mutant sequence was retained in vRNA recovered from these

7 leaves. PCRs originating from upper leaves of one p32KO-infected plant from each confirmed

8 systemic experiment were cloned and sequenced, and all 13 cDNAs contained the p32KO sequence

9 (data not shown). These results indicated that the p32KO mutation was very stable, and that the

10 characteristics of the infections were due to the lack of p32. p32KO was the only singly inoculated

11 MCMV mutant tested in this project that was recovered unchanged from systemic leaves (Table 1).

12 The low levels of virus and mild or absent symptoms may be directly due to defective replication of

13 p32KO as observed in protoplast inoculations, or p32 may have a role in enhancing viral movement or

14 interfering with plant defense.

153.4 Deletion of the amino-terminal portion of p50 decreases viral accumulation and/or spread in 16 plants

All 11 p50-AUG-inoculated plants developed WT-like systemic infections, but CP detection in inoculated leaves and systemic symptoms were delayed about six days. Restriction digests and sequencing of RT-PCRs from vRNA isolated from inoculated and systemic leaves at 21-30 dpi indicated that all systemic virions were partial revertants (Table 1). The p50 start codon was

21 regenerated, thereby removing the BsiWI restriction site (Fig. S2C) while leaving two additional p50-

22 AUG nt unchanged. A small fraction of RT-PCRs from inoculated leaves were digested by BsiWI

23 indicating that it could replicate in initially infected cells, as expected, but it was not possible to 
determine whether p50-AUG could move cell-to-cell or systemically without complementation by the WT-like revertant.

\section{5 p7a and p7b are required for cell-to-cell movement in plants}

The functions of p7a and p7b were tested on maize plants using transcripts from p7apTer, p7art, p7bKO, p7bpTer, and p7a/Cp, and some mutants were also tested in barley. No symptoms were observed, CP was not detected in any leaves, and no vRNA was detected in upper leaves for all five mutants (data not shown). Signals for (+) RNA from maize or barley plants inoculated with p7apTer, p7art, or p7a/CP could only be detected from inoculated leaves at 7 or 14 dpi at background levels similar to those of plants inoculated with the nonreplicating transcript $\Delta$ GDD using dot blot hybridization of total RNA, and no vRNA or sgRNA bands were detected on northern blots of total RNA from barley (20dpi) or maize (28 dpi) inoculated leaves for p7art and p7a/CP (Table 1 and data not shown). RT-PCR of vRNAs isolated from inoculated leaves at final time points for p7art or p7a/CP only produced detectable levels of a 432 bp DNA fragment when secondary PCR amplifications were performed as would be expected from accumulation of low levels of CP and virions only within initially infected cells (Table 1 and data not shown). Virus was detected by primary RT-PCR in inoculated leaves of all five p7bpTer and four of five p7bKO vRNA samples, possibly due to encapsidation of more vRNA since their CP accumulation levels are greater than other sgRNA ORF mutants (Fig. 4 and data not shown). Only three samples produced enough DNA for purification and direct sequencing. The single p7bpTer sample showed 100\% of the mutant input sequence while p7bKO samples showed one was 100\% mutant and the second contained the pseudorevertant GTG for the start codon (Table 2).

To determine whether cell-to-cell movement could be restored by supplying p7a or p7b in trans, mutant transcript combinations p7art + p7a/CP, p7bKO + p7apTer, or p7bpTer + p7apTer were coinoculated to seedlings. Five of the six plants inoculated with p7a/CP + p7art produced low but 
1 detectable levels of CP in the inoculated leaves at days 13, 20, or 26 but not at day 6 when WT signal

2 was detected, indicating that virus had slowly spread to additional cells and replicated (Table 1). Only

3 one plant had positive ELISA signals in the inoculated leaf at all later time points while the others gave

4 no signal on one or more later times suggesting that movement was slow and uneven. No CP was

5 detected in the newest leaves, and the plants were symptomless. RT-PCR of vRNAs isolated from all

6 inoculated leaves produced readily detectable DNA (Table 1). The presence of both mutants was

7 confirmed by single and double restriction digests with HpaI and $M f e$ I (Fig. S3). Cloned cDNAs from

8 two plants were sequenced. Seven plasmids each contained the p7a/CP or p7art sequence, while one

9 contained the $M f e$ I site of p7art but reverted to the WT stop codon for p7a (data not shown). For each

10 plant an equal number of cDNAs from both transcripts were recovered suggesting no preferential

11 accumulation of either mutant. Coinoculation with p7bKO + p7apTer or p7bpTer + p7apTer produced

12 delayed, mild systemic infections, and vRNA sequences recovered from systemic leaves were mixtures

13 of inoculating sequences and partial reversions (Table 1 and Suppl. data).

143.6 p31 enhances efficient systemic movement in plants

All five plants inoculated with p7bQ12N transcripts were symptomless but positive for CP in the inoculated leaf at $7 \mathrm{dpi}$ at readily detectable levels ( 20-100\% of WT range). This shows that the p7bQ12N infection had already spread cell-to-cell for further replication and CP synthesis, indicating that p31 was not required for cell-to-cell movement and that the conservative substitution in p7b did not significantly impair cell-to-cell movement. Systemic symptoms were delayed five to six days (Table 1). By 20 dpi, p7bQ12N plants showed symptoms that were milder than WT in older leaves and looked like WT in the newest leaf. RT-PCR followed by sequencing was performed for vRNAs isolated from inoculated and newest leaves at $20 \mathrm{dpi}$. Four inoculated leaf samples and three newest leaf samples showed a mixture of p7bQ12N and a pseudorevertant sequence, with the majority of the DNA derived from p7bQ12N in most samples while two newest leaf samples were $100 \%$ 
1 pseudorevertant (Table 2). The mutation removed the stop codon introduced downstream of the p7a

2 stop codon by changing nt 3235 from U to C while leaving both mutated bases, nt 3236 and 3238,

3 unchanged. The pseudorevertant would encode p7b with the same Q to $\mathrm{N}$ change of p7bQ12N which

4 further indicates that the conservative substitution did not impair p7b function. p31 had two residues

5 changed from WT: the pseudorevertant encodes glutamine-leucine while WT encodes serine-valine.

6 For p7bQ12N, the change from readily detectable infections in inoculated leaves to delayed appearance

7 of a pseudorevertant in systemic leaves shows that while p31 is not required for cell-to-cell movement,

8 it enhances long distance spread of MCMV. These results do not distinguish whether a stable mutation

9 that stops expression of the readthrough region can move to non-inoculated leaves, nor whether p31 is

10 directly involved with viral loading/unloading of phloem or if it acts as a systemic viral suppressor of

11 silencing (VSR).

123.7 CP is required for cell-to-cell movement in plants

Plants were inoculated with pCP-AUG or p7bMet to characterize the effect of stopping or decreasing full length CP production. In pCP-AUG the CP start codon was mutated to GTG, which can be used as a noncanonical start codon in some mRNAs, and initiation at the next GTG or ATG would produce $23 \mathrm{kDa}$ and $20 \mathrm{kDa}$ proteins, respectively (Fig. 3). If truncated $\mathrm{CP}$ is expressed it lacks the arginine-rich amino terminus shown to be required for packaging full length gRNA in the tombusvirus cucumber necrosis virus (Reade et al., 2010). Plants inoculated with pCP-AUG transcripts showed no symptoms and failed to produce a detectable infection in four experiments using maize (24 plants) and two experiments using susceptible barley (10 plants) (Table 1 and data not shown). Additionally, no signals from gRNA or sgRNA1 were detected on northern blots of total RNA from inoculated barley leaves (data not shown). Dot blot signals for (+) RNA could be detected in inoculated maize leaves at $7 \mathrm{dpi}$ at levels similar to those of plants inoculated with the nonreplicating transcript $\Delta \mathrm{GDD}$, and no RNA was detected at 14 and 21 dpi (data not shown). No CP was detected by ELISA in any tested leaf 
1 although the antiserum detects additional bands $~ 2$ and $5 \mathrm{kDa}$ smaller than WT CP on western blots of

2 protoplasts infected with MCMV (data not shown). This indicated that full length CP is required for

3 cell-to-cell movement of MCMV, and that a noncanonical GUG start codon at this location does not

4 function well in vivo. pCP-AUg and p7bMet transcripts produce similar low ELISA signals in

5 protoplasts (Scheets 2000, Fig. 4, and data not shown), but p7bMet produced systemic infections with

6 unusual local lesion-like symptoms in four of five plants, which suggests that MCMV may move cell-

7 to-cell as virions (Suppl. data).

8 4. Discussion

All tested viruses in this family have required both the upstream protein and the RdRp rt protein for replication. Coinoculation of p50-2stop and p111rt produced a low level of viral replication (Fig 2), and four tombusvirids have been shown to similarly trans complement replication (Batten et al., 2006; Molinari et al., 1998; Oster et al., 1998; White et al., 1995) while pelargonium flower break virus transcripts did not (Martinez-Turino and Hernández, 2010). Viruses from seven of the 12 monopartite tombusvirid genera are known to support trans replication of satellite RNAs, satellite viruses, and/or defective RNAs (Hull, 2014). This is the first report of trans-complementation from a genus that is not known to replicate a defective RNA, satellite RNA, or satellite virus.

Although the amino-terminal 23 residues of p50/p111 are not necessary for replication, its deletion reduced gRNA accumulation to about 1/4 WT levels in protoplasts and readily allowed a revertant to out-compete and spread systemically in plants. The overprinted portion of p50 may have a nonreplication function such as VSR. Alternatively, changes in RNA secondary structure in p50-AUG may have affected the relative translation initiation rates for p32 vs. the p50 ORF which may be deleterious for replication.

Similar to p32, the p23 ORF of hibiscus chlorotic ringspot virus (HCRSV) is overprinted within its ORF1 replication gene. p23 is required for long distance movement (Gao and Wong, 2013) and 
host-specific replication in kenaf but does not suppress silencing in the Nicotiana benthamiana 16c

assay (Liang et al., 2002). The three-fold decrease of gRNA and CP synthesis for p32KO transcripts in protoplasts suggests that p32 may have an auxiliary function in replication or may support infections by suppressing RNAi. p32KO mutations were stable in plants, and the slower spread and markedly reduced virus accumulation of p32KO are consistent with either function.

Mutations that alter the expression of upstream ORFs on sRNA1 can affect the accumulation of MCMV CP as seen with other tombusvirids (Castaño et al., 2009; Chkuaseli et al., 2015; Koh et al., 2006; Turina et al., 2000). For MCMV as well as panicoviruses (Scheets, 2013; Turina et al., 2000; Ziegler et al., 2009) and the proposed pelarspoviruses (Castaño et al., 2009; Scheets et al., 2015), the use of one sgRNA is supported by adjusting the relative strengths of translation initiation contexts (Nakagawa et al., 2008) and using a non-AUG start codon for MP2. MCMV is unique in having an sgRNA where three separate ORFs precede the CP AUG, with two ORFs overlapping the CP start codon.

All proteins encoded on sgRNA1 are associated with cell-to-cell and/or long distance viral spread. Without the ability to visualize spread via fluorescent protein expression, the results from these experiments do not rule out the possibility that lack of p7a, p7b, or CP may allow very limited spread below the level of detection of RNA or CP by the methods used in this work. These proteins function in trans, allowing accumulation and spread within the inoculated leaf. Complementation of MP1 and MP2 by coinoculating two mutant transcripts was successful for turnip crinkle virus in Arabidopsis (Cohen et al., 2000; Li et al., 1998), but not for melon necrotic spot virus on melon cotyledons (Genoves et al., 2006). MP1 and MP2 are sufficient for cell-to-cell movement of several tombusvirids (Genoves et al., 2006; Hacker et al., 1992; Jiwan et al., 2011; Molnar et al., 1997; Pantaleo et al., 1999), but others need additional sgRNA-encoded proteins such as p5' of beet black scorch virus (Yuan et al., 2006), CP for 
1 pelargonium line pattern virus (Castaño et al., 2009), or CP and its overprinted gene product for

2 HCRSV (Niu et al., 2014:Zhou et al., 2006). PMV movement requires all four sgRNA1-encoded

3 proteins (Turina et al., 2000).

Since p7bQ12N inoculated leaves had readily detectable CP at $7 \mathrm{dpi}$, and at $20 \mathrm{dpi}$ the majority of vRNA in inoculated leaves contained the mutant sequence, it strongly suggests that p31 is not required for cell-to-cell movement. The presence of pseudorevertant sequences dominating the vRNAs from upper leaves of p7bQ12N-inoculated plants indicates that p31 either directly or indirectly enhances or is required for systemic movement of MCMV (Tables 1 and 2).

A VSR may be encoded by one of the three unique MCMV coding regions. p32 has a WG motif while the readthrough region of p31 contains two GW motifs, and Argonaute-binding proteins often have a GW/WG motif, including some plant VSRs (Csorba et al., 2015). p32, p31, the readthrough region of $\mathrm{p} 31$, and $\mathrm{CP}$ were screened for possible VSR activity by testing for the ability to suppress silencing of the green fluorescent protein (GFP) gene when co-agroinoculated with a GFPexpressing plasmid on transgenic $N$. benthamiana expressing GFP (line 16c), but GFP expression was silenced for all four constructs (J. Schoelz and Scheets, unpublished data). Lack of silencing suppression by MCMV proteins with this assay may be due to incompatibility with a dicot system, although local silencing suppressors for maize necrotic streak virus (J. Schoelz, personal communication) and other Poaceae-limited viruses do function (Liu et al., 2012; Tatineni et al., 2012; Young et al., 2012). Systemic silencing suppressors are not readily detected by this assay (Brigneti et al., 1998; Senshu et al., 2011; Shimura et al., 2013; Yaegashi et al., 2007).

Experimental support that MCMV encodes a VSR comes from double-inoculation experiments with wheat streak mosaic virus (WSMV) (Scheets 1998). As well as the classic synergistic increase in MCMV accumulation in doubly-inoculated plants, the presence of MCMV increased the levels of WSMV under growing conditions that favor WSMV infection, and tripled the fraction of infected 
1 plants in unfavorable growing conditions (Scheets, 1998). Natural infection VSR assays use maize or

2 soybean varieties that express the dominant inhibitory chalcone synthase (CS) allele, which acts via

3 endogenous RNA silencing in maize leaves (Della Vedova et al., 2005) or soybean seed coats (Senda et

4 al., 2004), respectively. Infections of plants with viruses from genera encoding VSRs that function in

$5 \quad N$. benthamiana line 16c suppress the silencing and produce anthocyanin, while infection of the

6 silenced maize line with MCMV does not (Della Vedova et al., 2005; Senda et al., 2004), suggesting

7 that MCMV does not have an effective local VSR.

8 5. Conclusions

Maize chlorotic mottle virus is a unique member of the family Tombusviridae. By using mutated versions of MCMV lacking one or two expressible ORFs in maize protoplast and plant inoculations it was shown that five of the proteins have functions similar to the homologous proteins in related tombusvirid genera. Two unique ORFs encoded in MCMV, both of which overlap other ORFs, encode functional proteins. p32 enhances accumulation in protoplasts and enhances symptoms and accumulation in plants, while p31 is required for efficient systemic movement. BLASTp searches for both encoded proteins produce matches with scores less than 42 , and the functions of the match proteins do not readily suggest a similar function for the MCMV proteins. The unique amino-terminal region of p50 that overlaps the p32 ORF may also have additional functions. Further work is needed to determine how these proteins provide their selective advantage for MCMV infections in maize.

\section{Acknowledgements}

The author thanks Carri Bode, Mike Byrom, Amy Crain, Brian Decocq, Jeff Dill, Bryan Haggard, Nathan Lassiter, Chris Royer, Cynthia Stoner, Sandi Ulrich, and Brad White for laboratory assistance, and Ulrich Melcher and Jim Schoelz for manuscript review. I also thank the OSU

Recombinant DNA/Protein Core Facility for DNA sequencing. Funding was provided by Pioneer Hi- 
Bred International, United States Department of Agriculture National Research Initiative (93-373039036), and Oklahoma Center for the Advancement of Science and Technology (HN5-007).

\section{References}

Batten, J., Turina, M., Scholthof, K.-B., 2006. Panicovirus accumulation is governed by two membrane-associated proteins with a newly identified conserved motif that contributes to pathogenicity. Virol. J. 3, 12.

Brigneti, G., Voinnet, O., Li, W. X., Ji, L. H., Ding, S. W., Baulcombe, D. C. 1998. Viral pathogenicity determinants are suppressors of transgene silencing in Nicotiana benthamiana. EMBO J. 17, 67396746.

Castaño, A., Ruiz, L., Hernández, C., 2009. Insights into the translational regulation of biologically active open reading frames of pelargonium line pattern virus. Virology 386, 417-426.

Chkuaseli, T., Newburn, L. R., Bakhshinyan, D., White, K. A. 2015. Protein expression strategies in tobacco necrosis virus-D. Virology 486, 54-62.

Cohen, Y., Gisel, A., Zambryski, P.C., 2000. Cell-to-cell and systemic movement of recombinant green fluorescent protein-tagged turnip crinkle viruses. Virology 273, 258-266.

Csorba, T., Kontra, L., Burgyan, J., 2015. Viral silencing suppressors: Tools forged to fine-tune hostpathogen coexistence. Virology 479-480, 85-103.

Della Vedova, C.B., Lorbiecke, R., Kirsch, H., Schulte, M.B., Scheets, K., Borchert, L.M., Scheffler, B.E., Wienand, U., Cone, K.C., Birchler, J.A., 2005. The dominant inhibitory chalcone synthase allele C2-Idf (Inhibitor diffuse) from Zea mays (L.) acts via an endogenous RNA silencing mechanism. Genetics 170, 1989-2002.

Gao, R., Wong, S.M., 2013. Basic amino acid mutations in the nuclear localization signal of hibiscus chlorotic ringspot virus p23 inhibit virus long distance movement. PLoS One 8, e74000.

Genoves, A., Navarro, J.A., Pallas, V., 2006. Functional analysis of the five melon necrotic spot virus genome-encoded proteins. J. Gen. Virol. 87, 2371-2380.

Goldberg, K.B., Brakke, M.K., 1987. Concentration of maize chlorotic mottle virus increased in mixed infections with maize dwarf mosaic virus, strain B. Phytopathology 77, 162-167.

Hacker, D.L., Petty, I.T.D., Wei, N., Morris, T.J., 1992. Turnip crinkle virus genes required for RNA replication and virus movement. Virology 186, 1-8.

Hull, R., 2014. Plant Virology (5th Edition). 2014. Academic Press, Boston.

Iwakawa, H.-O., Mizumoto, H., Nagano, H., Imoto, Y., Takigawa, K., Sarawaneeyaruk, S., Kaido, M., Mise, K., Okuno, T., 2008. A viral noncoding RNA generated by cis-element-mediated protection 
against 5' $\rightarrow$ 3' RNA decay represses both cap-independent and cap-dependent translation. J. Virol. 82, $10162-10174$.

Jiwan, S.D., Wu, B., White, K.A., 2011. Subgenomic mRNA transcription in tobacco necrosis virus. Virology 418, 1-11.

Koh, D.C.-Y., Wang, X., Wong, S.-M., Liu, D.X., 2006. Translation initiation at an upstream CUG codon regulates the expression of hibiscus chlorotic ringspot virus coat protein. Virus Res. 122, 35-44.

Kunkel, T.A., Roberts, J.D., Zakour, R.A., 1987. Rapid and efficient site-specific mutagenesis without phenotypic selection, Methods Enzymol. Academic Press, pp. 367-382.

Li, W.-Z., Qu, F., Morris, T.J., 1998. Cell-to-cell movement of turnip crinkle virus is controlled by two small open reading frames that function in trans. Virology 244, 405-416.

Liang, X.-Z., Lucy, A.P., Ding, S.-W., Wong, S.-M., 2002. The p23 protein of hibiscus chlorotic ringspot virus is indispensable for host-specific replication. J. Virol. 76, 12312-12319.

Liu, Y., Zhai, H., Zhao, K., Wu, B., Wang, X., 2012. Two suppressors of RNA silencing encoded by cereal-infecting members of the family Luteoviridae. J. Gen. Virol. 93, 1825-1830.

Martinez-Turino, S., Hernández, C., 2010. Identification and characterization of RNA-binding activity in the ORF1-encoded replicase protein of pelargonium flower break virus. J. Gen. Virol. 91, 30753084.

Molinari, P., Marusic, C., Lucioli, A., Tavazza, R., Tavazza, M., 1998. Identification of artichoke mottled crinkle virus (AMCV) proteins required for virus replication: complementation of AMCV p33 and p92 replication-defective mutants. J. Gen. Virol. 79, 639-647.

Molnar, A., Havelda, Z., Dalmay, T., Szutorisz, H., Burgyan, J., 1997. Complete nucleotide sequence of tobacco necrosis virus strain DH and genes required for RNA replication and virus movement. J. Gen. Virol. 78, 1235-1239.

Nakagawa, S., Niimura, Y., Gojobori, T., Tanaka, H., Miura, K., 2008. Diversity of preferred nucleotide sequences around the translation initiation codon in eukaryote genomes. Nucleic Acids Res. 36, 861871.

Niblett, C.L., Claflin, L.E., 1978. Corn lethal necrosis - a new virus disease of corn in Kansas. Plant Dis. 62, 15-19.

Niu, S., Gil-Salas, F.M., Tewary, S.K., Samales, A.K., Johnson, J., Swaminathan, K., Wong, S.M., 2014. Hibiscus chlorotic ringspot virus coat protein is essential for cell-to-cell and long-distance movement but not for viral RNA replication. PLoS One 9, e113347.

Nutter, R.C., Scheets, K., Panganiban, L.C., Lommel, S.A., 1989. The complete nucleotide sequence of the maize chlorotic mottle virus genome. Nucleic Acids Res. 17, 3163-3177. 
Oster, S.K., Wu, B., White, K.A., 1998. Uncoupled expression of p33 and p92 permits amplification of tomato bushy stunt virus RNAs. J. Virol. 72, 5845-5851.

Pantaleo, V., Grieco, F., Castellano, M.A., Martelli, G.P., 1999. Synthesis of infectious transcripts of olive latent virus 1: genes required for RNA replication and virus movement. Arch. Virol. 144, 10711079.

Reade, R., Kakani, K., Rochon, D.A., 2010. A highly basic KGKKGK sequence in the RNA-binding domain of the cucumber necrosis virus coat protein is associated with encapsidation of full-length CNV RNA during infection. Virology 403, 181-188.

Riviere, C.J., Rochon, D.A.M., 1990. Nucleotide sequence and genomic organization of melon necrotic spot virus. J. Gen. Virol. 71 1887-1896.

Rochon, D., Rubino, L., Russo, M., Martelli, G.P., Lommel, S., 2011. Tombusviridae, in: King, A.M.Q., Adams, M.J., Carstens, E.B., Lefkowitz, E.J. (Eds.), Virus taxonomy: classification and nomenclature of viruses: Ninth Report of the International Committee on Taxonomy of Viruses. Elsevier, San Diego, pp. 1111-1138.

Scheets, K., 1998. Maize chlorotic mottle machlomovirus and wheat streak mosaic rymovirus concentrations increase in the synergistic disease corn lethal necrosis. Virology 242, 28-38.

Scheets, K., 2000. Maize chlorotic mottle machlomovirus expresses its coat protein from a 1.47-kb subgenomic RNA and makes a 0.34-kb subgenomic RNA. Virology 267, 90-101.

Scheets, K., 2013. Infectious transcripts of an asymptomatic panicovirus identified from a metagenomic survey. Virus Res. 176, 161-168.

Scheets, K., Jordan, R., White, K.A., Hernández, C., 2015. Pelarspovirus, a proposed new genus in the family Tombusviridae. Arch. Virol., 160:2385-2393.

Scheets, K., Khosravi-Far, R., Nutter, R.C., 1993. Transcripts of a maize chlorotic mottle virus cDNA clone replicate in maize protoplasts and infect maize plants. Virology 193, 1006-1009.

Senda, M., Masuta, C., Ohnishi, S., Goto, K., Kasai, A., Sano, T., Hong, J.S., MacFarlane, S., 2004. Patterning of virus-infected Glycine max seed coat is associated with suppression of endogenous silencing of chalcone synthase genes. Plant Cell 16, 807-818.

Senshu, H., Yamaji, Y., Minato, N., Shiraishi, T., Maejima, K., Hashimoto, M., Miura, C., Neriya, Y., Namba, S. 2011. A dual strategy for the suppression of host antiviral silencing: two distinct suppressors for viral replication and viral movement encoded by potato virus M. Journal of Virology 85(19), 10269-10278.

Shimura, H., Masuta, C., Yoshida, N., Sueda, K. Suzuki, M. (2013). The 2b protein of asparagus virus 2 functions as an RNA silencing suppressor against systemic silencing to prove functional synteny with related cucumoviruses. Virology 442(2), 180-188. 
Tatineni, S., Qu, F., Li, R., Jack Morris, T., French, R., 2012. Triticum mosaic poacevirus enlists P1 rather than HC-Pro to suppress RNA silencing-mediated host defense. Virology 433, 104-115.

Turina, M., Desvoyes, B., Scholthof, K.-B.G., 2000. A gene cluster encoded by panicum mosaic virus is associated with virus movement. Virology 266, 120-128.

Wangai, A.W., Redinbaugh, M.G., Kinyua, Z.M., Miano, D.W., Leley, P.K., Kasina, M., Mahuku, G., Scheets, K., Jeffers, D., 2012. First report of maize chlorotic mottle virus and maize lethal necrosis in Kenya. Plant Dis. 96, 1582.

White, K.A., Skuzeski, J.M., Li, W., Wei, N., Morris, T.J., 1995. Immunodetection, expression strategy and complementation of turnip crinkle virus p28 and p88 replication components. Virology 211, 525534.

Xia, Z., Zhao, Z., Chen, L., Li, M., Zhou, T., Deng, C., Zhou, Q., Fan, Z., 2016. Synergistic infection of two viruses MCMV and SCMV increases the accumulations of both MCMV and MCMV-derived siRNAs in maize. Scientific Reports 6, 20520.

Yaegashi, H., Takahashi, T., Isogai, M., Kobori, T., Ohki, S., Yoshikawa, N. (2007). Apple chlorotic leaf spot virus $50 \mathrm{kDa}$ movement protein acts as a suppressor of systemic silencing without interfering with local silencing in Nicotiana benthamiana. J. Gen. Virol. 88, 316-324.

Young, B. A., Stenger, D. C., Qu, F., Morris, T. J., Tatineni, S., French, R. (2012). Tritimovirus P1 functions as a suppressor of RNA silencing and an enhancer of disease symptoms. Virus Res. 163, 672677.

Yuan, X., Cao, Y., Xi, D., Guo, L., Han, C., Li, D., Zhai, Y., Yu, J., 2006. Analysis of the subgenomic RNAs and the small open reading frames of beet black scorch virus. J. Gen. Virol. 87, 3077-3086.

Zhou, T., Fan, Z.F., Li, H.F., Wong, S.M., 2006. Hibiscus chlorotic ringspot virus p27 and its isoforms affect symptom expression and potentiate virus movement in kenaf (Hibiscus cannabinus L.). Mol. Plant-Microbe Interact. 19, 948-957.

Ziegler, A., Cowan, G., Torrance, L., 2009. Comparative sequence analysis and serological and infectivity studies indicate that Cocksfoot mild mosaic virus is a member of the genus Panicovirus. Arch. Virol. 154, 1545-1549.

\section{Figure Legends}

Fig. 1. Genome maps of WT and mutant plasmid inserts. Heavy lines represent genomic RNA, and boxes mark the coding regions and their relative reading frames. The locations of sgRNA1 and sgRNA2 are shown for WT. The sizes in $\mathrm{kDa}$ of the encoded proteins are shown, and the leaky stop codons are marked. Dashed lines mark a non-AUG start codon. Gray boxes denote smaller ORFs produced by start or stop codon mutations. The deleted region of $\Delta \mathrm{GDD}$ is bordered by wavy lines. 
1 Fig. 2. Viral RNA and CP accumulation at 48 hpi in BMS protoplasts inoculated with WT or mutant

2 transcripts with start codon or stop codon changes in the 5' half of the genome. The upper northern blot

3 represents equal aliquots of total RNA/lane, while the lower blot was made from a second gel loaded

4 with duplicate samples of the left five lanes and a dilution representing 1/20 as much initial sample for

5 the last three lanes. The 18S rRNA photograph is from the upper gel. The lane representing

6 inoculation with equal amounts of p50-2stop and p111rt is labelled as Both. Relative CP accumulation

7 was calculated from protoplast PAS-ELISA data for three (Both) or 5 to 9 separate inoculation

8 experiments and represents mean \pm standard error.

9 Fig. 3. Genome maps of MCMV sgRNA1 and sgRNAs representing versions of pMCM41 encoding 10 mutations within the sgRNA1 region. Solid triangles on WT sgRNA1 mark AUG codons within the

11 ORFs (open boxes) while the open triangle and dashed line marks the CUG start codon for p7b. ORFs 12 are shown in relative reading frames. Gray boxes are smaller ORFs produced by start or stop codon 13 mutations, and the asterisks in sg7bQ12N and sg7bMet indicate coding changes in overlapping ORFs.

14 Parentheses enclose former names of four mutants described in Scheets, 2000.

15 Fig. 4. Viral RNA and CP accumulation at 48 hpi in BMS protoplasts inoculated with WT or mutant transcripts with start codon or stop codon changes in the sgRNA1 region of the genome. Samples (0, 1724 , and $48 \mathrm{hpi}$ ) from a single inoculation experiment were separated on a single gel and analyzed by northern hybridization (Fig S1). Only the 48 hpi lanes are shown here along with the corresponding

19 18S rRNA bands of the ethidium bromide-stained gel. Samples represent equal aliquots of total RNA/lane. Relative CP accumulation was calculated from protoplast PAS-ELISA data at 48 hpi and 21 represents mean \pm standard error of duplicate protoplast inoculations.

22 Fig. 5. Virus accumulation at $28 \mathrm{dpi}$ in the inoculated leaf and each upper leaf from infected plants 23 inoculated with transcripts of p32KO (gray bars) or WT (open bars). Bars represent means with 
1 standard errors for four p32KO plants and three WT plants. All but one plant of each type were at the 2 10-leaf stage.

3 